\title{
HASARD VÉCU ET NÉCESSITÉ SUBIE DANS LE ANTON REISER DE KARL PHILIPP MORITZ
}

\author{
Anne LAGNY
}

\begin{abstract}
RÉsumÉ : La distinction entre hasard « littéraire » et hasard vécu (E. Köhler) permet de dégager l'originalité du roman autobiographique de Karl Philipp Moritz. L'étude du motif du hasard dans le livre II d'Anton Reiser montre la mise en place d'un dispositif narratif caractérisé par le refoulement du traitement « littéraire » du hasard et le maintien de la perspective du hasard comme catégorie du vécu. Les hasards heureux et malheureux ne fondent pas ici la nécessité littéraire d'un destin exemplaire, mais sont l'occasion d'éclairer la double détermination, extérieure et intérieure, qui affecte l'existence du personnage, vécue sous le signe de la contingence radicale. C'est dans cette obscurité première que l'analyse psychologique opère, non pour restaurer l'horizon de la Providence, mais pour dégager les leçons générales d'une existence, et donner, à ce moment, à son héros la consistance d'un personnage de roman.
\end{abstract}

MOTS-CLÉS : hasard, contingence, narration, autobiographie, analyse psychologique.

ABSTRACT: The distinction between literary chance and chance as experience (E. Köhler) allows the originality of Moritz's autobiographical novel to be disengaged. This study of the motif of chance in book II of Anton Reiser shows the establishment of a narrative method characterized by repressing the literary treatment of chance and maintaining of chance as a category of lived experience. In this text, the fortunate and unfortunate chances do not found the literary necessity of an exemplary destiny, but are the occasion for throwing light on the double determination, exterior and interior, that affects the character's existence, an existence that is experienced under the sign of a radical contingency. It is in this first obscurity that psychological analysis operates, not to establish the horizon of Providence, but to disengage the general lessons of an existence, and to give to its hero, at this moment, the consistency of a novelistic character.

KEYWORDS : chance, contingency, narrative, autobiography, psychological analysis.

Revue de synthèse : $4^{\mathrm{e}} \mathrm{S}$. $\mathrm{N}^{\mathrm{os}} 3-4$, juil.-déc. 1996, p. 461-483. 
ZuSAMmENFASSUNG: Anhand von Köhlers Unterscheidung zwischen "literarischem " und " erlebtem » Zufall soll die Eigenart von Moritz' autobiographischem Roman in der Spannung zwischen Biographie und Roman verdeutlicht werden. Die Detailanalyse des Zufallmotivs im II. Buch zeigt, daß bei Moritz wohl ein Ansatz zur literarischen Gestaltung des Zufalls zu finden ist, daß aber die Aufrechterhaltung des Zufalls als Erlebniskategorie im Romangeschehen allein seinem Vorhaben gerecht werden kann. Der Zufall fungiert dementsprechend nicht mehr als Erfullungsgehilfe eines exemplarischen Schicksals (wie z. B. in den Leiden des jungen Werthers oder in Blanckenburgs Modell), beziehungsweise als Vermittler der literarischen Notwendigkeit, sondern dient vornehmlich dazu, die doppelte (innere so wie äußere) Determination der Romanfigur zu beleuchten, dessen Leben im Zeichen der Kontigenz steht. Hier setzt die psychologische Analyse an und dadurch wird der Romanfigur bei allem Partikularen doch eine gewisse Exemplarität verliehen, wenn auch die Providenz endgültig aus dem Blickfeld gerät.

STICHWÖRTER : Zufall, Kontingenz, Erzählung, Autobiographie, psychologische Analyse.

Anne LAGNY, née en 1955, agrégée d'allemand et docteur, est maître de conférences à l'université de Lille III. Elle travaille sur le xviII ${ }^{e}$ siècle allemand, en particulier sur Lessing. Adresse : Université de Lille III, BP 149, 59653 Villeneuve-d'Ascq Cedex. 
Je dédie ce texte à mes étudiants de Lille. C'est avec eux, à partir de leurs questions que mon travail a commencé.

Dans son étude consacrée au hasard en littérature ${ }^{1}$, Erich Köhler distingue entre le hasard comme «catégorie du vécu» et le « hasard littéraire », c'est-à-dire tel que l'écrivain le fait intervenir dans sa narration pour infléchir le parcours de ses personnages. Traité « littérairement », le hasard perd son caractère particulier pour acquérir un degré de généralité et de nécessité satisfaisant aux exigences de l'œuvre, laquelle, selon le mot de Flaubert, doit être «plus logique que le hasard des choses». Le romancier qui se donne pour tâche de tendre la ligne du destin d'un personnage et d'en faire apparaître la nécessité est amené à bannir ce qui, dans la réalité brute qui l'entoure, se présente comme éparpillé, fragmentaire, et à ne retenir que les matériaux servant son dessein. Dans cette recomposition à partir d'éléments triés et concentrés, le hasard n'intervient plus comme facteur d'encombrement et d'illisibilité de l'existence, mais comme maillon d'une concaténation dans laquelle se lit la «nécessité » d'un destin littéraire. Il n'y a pas de hasard gratuit dans l'œuvre. Là où l'auteur choisit de le faire intervenir, il fonctionne comme mode d'apparition de la nécessité qui fait le sens de l'œuvre. Le hasard d'une rencontre inattendue ou d'un rendezvous manqué, d'une lettre non reçue ou d'une correspondance découverte, même s'il joue à la frontière de la vraisemblance, devient l'occasion de révéler des déterminations (sociales, psychologiques) qui sans lui, seraient restées cachées, et dont la figuration constitue le sens de l'œuvre.

La distinction entre ces deux catégories du hasard vécu et du hasard littéraire nous paraît très utile pour aborder l'étude du roman autobiographique de Karl Philipp Moritz, Anton Reiser, sous l'angle particulier de l'ambivalence d'un projet « romanesque » en un sens nouveau. Moritz donne en effet son œuvre pour un « roman psychologique », dans lequel il s'attache à restituer, par l'introspection, la vie intérieure de son personnage à partir de données (auto-)biographiques. La perspective narrative ainsi définie inclut le point de vue de l'individu immergé dans la réalité, sans distance réflexive assignant un sens clair aux événements, au moins dans un premier temps. Elle réserve donc la possibilité de figurer le hasard comme « catégorie du vécu » - une dimension que l'auteur plus soucieux de « littérature »

1. Erich KÖHLER, Der literarische Zufall, das Mögliche und die Notwendigkeit, Munich, Fink Verlag, 1973, trad. franç. Éliane KaufHolz, Le Hasard en littérature, le Possible et la Nécessité, Paris, Klincksieck, 1986. 
tendrait justement à exclure pour construire son roman -, ce qui implique réaménagement, sinon bouleversement du projet romanesque. Comment figurer la perspective du hasard vécu, dans sa particularité et son " insignifiance " ? Comment la concilier avec l'exigence d'une forme de généralité et de nécessité sans lesquelles l'œuvre ne peut prétendre à un niveau de représentativité ? Cette question nous introduit au cœur de la problématique du « roman psychologique » telle que Moritz la définit dans les préfaces qu'il a données aux quatre livres d'Anton Reiser.

Comme le souligne Moritz dans l'une de ses préfaces, le maintien de la perspective du héros plongé dans l'immédiateté de ce qu'il vit ne va pas sans malaise pour le lecteur. Celui-ci, confronté à une accumulation de détails infimes et de «petits faits », éprouve quelque peine à saisir le sens général de l'œuvre et sa nécessité littéraire. Un certain nombre de témoignages, correspondant à la première phase de la réception du roman, font état d'une déception devant l'insignifiance d'une pareille existence, ainsi représentée. La réflexion irrévérencieuse de Heine est sans doute l'expression la plus lapidaire de cette incompréhension fondamentale : parlant d'une œuvre largement oubliée à son époque (1826), il caractérise le roman comme "l'histoire de quelques centaines de thalers qui manquaient à l'auteur, ce qui a fait de sa vie une suite de privations et de renoncements $»^{2}$. Vu par Heine avec la distance de l'ironie, le roman de Moritz se ramène à une histoire banale de la condition humaine commune, digne tout au plus d'une mention fugitive. Quel sens y trouver, en effet ? Cette histoire pitoyable d'une vie inaccomplie, réduite à l'anecdote, n'a même pas le don d'émouvoir le lecteur. Non seulement Heine refuse de considérer la misère matérielle comme détermination suffisante d'un véritable destin, mais encore il épingle les prétentions injustifiées de l'auteur à la publicité. Celui-ci ne va-t-il pas s'imaginer que son destin d'épigone de Gothe peut susciter l'intérêt? Pour Heine, le cas est clair. Entre l'auteur de Werther et celui d'Anton Reiser, le lecteur (et avec lui la postérité) a choisi. Le premier nous intéresse et nous retient, le second est quantité négligeable, comme en témoigne d'ailleurs la fortune du roman, tombé dans un oubli mérité.

2. Cf. la citation complète extraite des Reisebilder, "Die Nordsee ", Sämtliche Werke, 6, Düsseldorf, Hoffmann \& Campe, 1973, p. 287-288. C'est la version française, due à Heine, du texte p. 145, note p. 744 : "Ce roman déjà complètement oublié, contenait l'histoire de l'auteur, ou plutôt l'histoire de quelques [sic] cent thalers que l'auteur n'avait pas, et par le manque desquels sa vie entière ne devint qu'une suite de privations et de désenchantements. Cependant les prétentions du malheureux n'étaient rien moins qu'immodestes; par exemple, [encore jeune homme] il avait le désir de se rendre à Weimar et de se faire le domestique de l'auteur de Werther, à quelques conditions que ce fût, dans le seul but de vivre dans le voisinage de celui qui, d'entre tous les hommes sur terre, avait produit sur son âme la plus forte impression. " 
Ces réflexions de Heine sont à rapprocher d'une recension anonyme parue à la sortie du livre I d'Anton Reiser, à laquelle Moritz répond dans la préface au livre II :

«Ce que nous critiquerons dans son plan, c'est l'état et la situation où il fait naître et grandir ses héros. Comme Monsieur Moritz écrit tout de même pour des gens qui savent lire, c'est-à-dire qui ne sont pas artisans ou autres gens du même acabit, il aurait dû éviter des scènes dont les détails ne peuvent éveiller chez les lecteurs que l'ennui, au lieu de la sympathie. Il rendrait aussi son livre plus intéressant et plus instructif s'il voulait donner à ses observations un cadre plus clair et, de façon générale, une anatomie et une météorologie de l'âme plus philosophique qu'historique ${ }^{3}$.

Le recenseur avoue plus tard que l'épithète de roman l'a induit en erreur et conseille à Moritz d'intituler son livre «Bekenntnisse» (Confessions).

Ce faisant, le recenseur met le doigt sur la difficulté propre à l'entreprise de Moritz : arriver à donner une forme à ce qui n'en a pas, à ce qui n'a pas d'intérêt manifeste. La trivialité du parcours d'Anton Reiser est mise directement en rapport avec la condition sociale du héros : le « quatrième état » dans lequel il est né n'offre pas de possibilités, semble-t-il, de vivre une histoire « intéressante », susceptible de fournir la matière d'un roman. Pour un bourgeois cultivé, peu encombré par les problèmes matériels, la véritable vie - ou le destin du personnage - n'est pas conditionnée par les soucis d'argent ${ }^{4}$. Les tribulations d'un représentant du « quatrième état » sont tout au plus admises à titre de document (auto-)biographique.

3. Cette recension, parue dans la Gothaische Gelehrte Zeitung en 1785, est citée in Karl Philipp Moritz, Werke, 3 vol., $1^{\text {re }}$ éd. 1981, Francfort, Insel Verlag, 1993, I, « Autobiographische und poetische Schriften », p. 573: "Was wir an seinem Plane auszusetzen haben, ist der Stand und die Situationen, worin er seine Helden geboren werden und aufwachsen läßt. Da Hr. Moritz doch für Leute schreibt, die lesen, das heißt nicht für Handwerker u. dgl., so hätte er sich auch für [vor] Szenen hüten sollen, deren Details seinen Lesern statt Teilnahme Langeweile erregen müssen. Gewiß auch würde er sein Buch interessanter und lehrreicher machen, wenn er den Beobachtungen einen sichtbareren Zusammenhang und überhaupt mehr eine philosophische als historische Anatomie und Meteorologie der Seele geben wollte " (c'est moi qui souligne). Le terme de «historisch» est à prendre au sens où on le trouve par exemple dans « histoire naturelle », et renvoie à une collection de faits.

4. Cet aspect social de la critique mériterait un développement particulier, qui déborde le cadre de cette étude. La comparaison entre Werther et Anton Reiser, par exemple, serait intéressante à développer de ce point de vue. Citons encore l'introduction rédigée par Gœthe en 1822 à l'autobiographie d'un domestique, éditée sous le titre : « Der deutsche Gil Blas »,

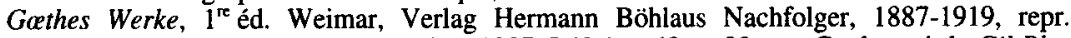
Munich, Deutscher Taschenbuch Verlag, 1987, I.42.1, t. 48, p. 88 sqq. Gœthe voit le Gil Blas de Lesage comme une cuvre de l'art («Kunstwerk»), alors que le texte qu'il caractérise comme « la Bible des domestiques et des artisans » est une cuvre de la nature (« Naturwerk $\gg)$, instructive par le détail, mais dénuée de la représentativité supérieure du roman, qui présente des « résultats ». On retrouve donc chez Gathe, autrement, le clivage entre le biographique et le romanesque qui est une des données du projet de Moritz. 
Ce qui nous intéresse ici plus immédiatement, c'est la tension qui apparaît dans cette critique entre le «biographique " (l'histoire sans le cadre «philosophique" qui lui donnerait forme et sens) et le "romanesque ". C'est sur ce point précis que Moritz se défend de la critique, sans s'arrêter aux préjugés sociaux implicites du recenseur anonyme. Dans ses préfaces aux deux premiers livres, il souligne la concurrence de deux intentions: écrire un roman, écrire une « histoire intérieure » étayée par des matériaux (auto-)biographiques. À ce stade, il est clair que Moritz s'appuie plus sur l'aspect biographique que sur l'aspect romanesque pour introduire son cuvre auprès de lecteurs traditionnels ${ }^{5}$. Ce faisant, il réintroduit le critère de la véracité, la caution du documentaire, mais aussi, et fortement, la dimension du particulier.

La perspective définie par Moritz comme celle de l'histoire intérieure est celle de l'individu immergé dans la confusion de l'immédiateté. À ce premier niveau de perception, les petits faits de l'existence apparaissent insignifiants et décousus ${ }^{6}$. Seul l'examen rétrospectif, le retour de la conscience, permet de les éclairer en les coordonnant entre eux. Dans la perspective limitée d'Anton, de l'enfant, puis de l'adolescent dont la faculté de penser n'est pas encore exercée, il est impossible d'assigner un sens clair à ce qui survient. Pour Moritz, il importe de conserver un temps la perspective de la conscience obscure, sans imposer prématurément une interprétation des faits. Son intention est donc bien d'intégrer le hasard comme catégorie du vécu en écartant, au moins provisoirement, la solution d'un traitement « littéraire " tel que le définit Erich Köhler : c'est-à-dire au sens où l'accident qui survient, et jusqu'à la tuile qui tombe sur la tête du héros, perd son caractère particulier pour s'insérer dans la logique d'un tout, celui de la figuration littéraire et de sa nécessité propre, qui fait le sens de l'œuvre ${ }^{7}$. Au point que sans ce « hasard " littéraire, le roman n'est pas pensable. À charge pour l'auteur de choisir le hasard qui manifestera la logique de la destinée du personnage comme forme de nécessité littéraire.

La comparaison entre Anton Reiser et Werther, deux œuvres contemporaines, fera mieux sentir la différence de nature entre le hasard vécu et le

5. K. P. Moritz, dans ses premières préfaces, esquisse négativement « l'horizon d'attente » du lecteur de romans (l'intérêt des événements, la diversité des caractères) pour mieux marquer les caractères propres de son cuvre et prévenir ainsi la déception probable du public devant l'insignifiance apparente des petits faits, et la concentration de l'intérêt sur une figure.

6. K. P. Moritz, Anton Reiser, éd. Wolfgang Martens, Stuttgart, Reclam, 1986 (cité par la suite comme Anton Reiser), p. 122 : «Zwecklosigkeit, abgerißne Fäden, Verwirrung, Nacht und Dunkelheit. "

7. E. Köhler cite le cas du roman de Fontane, Effi Briest : le hasard qui amène le mari à découvrir la correspondance adultère n'est pas intéressant en tant que tel, mais par la chaîne d'événements qui va en résulter pour constituer la destinée d'Effi Briest, et le roman. 
hasard littéraire, du point de vue de la figuration. Dans le roman de Gœethe, l'occasion de la rencontre avec Lotte est fortuite et signalée comme telle :

« Je t'ai écrit récemment que j'avais fait la connaissance du bailli S., et qu'il m'avait prié de lui rendre bientôt visite dans son ermitage, ou plus exactement dans son petit royaume. Je négligeai son offre, et je n'y serais peut-être jamais allé, si le hasard ne m'avait pas découvert le trésor qui est caché dans cette région paisible ${ }^{8}$.

Le hasard intervient par surprise, comme agent actif, pour infléchir le parcours du héros et l'orienter vers l'accomplissement de la passion malheureuse. Il précipite le destin de Werther et le fait apparaître tout d'abord sous la forme des célèbres rubans roses de Lotte. On notera que l'occasion de la rencontre décisive est une visite de courtoisie que le héros distrait est bien près d'oublier. Le destin de Werther, un instant, est à la merci d'une négligence qui le ferait passer à côté de l'événement destiné à bouleverser son existence.

Werther, étrangement, ne s'étonne pas de cet accident fortuit, ce qui implique une distribution claire des fonctions : ce n'est pas au héros qui vit l'histoire de s'arrêter à ce détail, agencé par une instance supérieure, celle qui ordonne la narration. Le lecteur n'a pas davantage le loisir de s'en émerveiller. En l'espace d'une phrase, le hasard devient signifiant, il est interprété par le héros comme l'occasion de la découverte d'un trésor, instantanément converti en nécessité de la passion. Il contribue de manière déterminante à la configuration du destin du héros et fonctionne donc comme « mode d'apparition de la nécessité », selon le terme de Köhler : la rencontre de Lotte détermine fatalement le destin de Werther.

Si nous nous tournons maintenant vers Anton Reiser, il nous semble que la fin du livre I et le début du livre II, par certains aspects, offrent un exemple d'amorce, mais d'amorce seulement, d'une semblable utilisation « littéraire » du hasard. L'étude attentive de ce passage montrera que cette perspective est intenable à long terme, sous peine de dénaturer le projet de Moritz. Celui-ci exige en effet, pour garder sa cohérence, le maintien de la perspective du hasard vécu. Rappelons brièvement la situation : le père d'Anton ne voit aucune objection à ce qu'il fasse des études, mais se refuse à lui fournir les moyens financiers nécessaires. Anton est impuissant, ses résolutions héroïques, calquées sur les exemples antiques, se heurtent à l'impossibilité concrète de travailler et d'étudier. Dans cette impasse, une circonstance fortuite intervient pour changer la situation :

8. Cf. Gothes Werke, op. cit. supra n. 4, $1^{\text {re }}$ éd. Weimar, I, 19, t. 22, p. 25 : «Ich schrieb dir neulich, wie ich den Amtmann S. habe kennen lernen, und wie er mich gebeten habe, ihn bald in seiner Einsiedelei, oder vielmehr seinem kleinen Königreiche zu besuchen. Ich vernachlässigte das, und wäre vielleicht nie hingekommen, hätte mir der Zufall nicht den Schatz entdeckt, der in der stillen Gegend verborgen liegt. 》 
« Aucune perspective sérieuse ne s'offrait donc à Anton pour le cas où il aurait entrepris des études et il aurait sans doute dû se résoudre, en fin de compte, à apprendre quelque métier manuel si, contre toute attente, un détail d'apparence insignifiante n'avait donné à sa destinée et à toute son existence une autre direction. »

Le livre II commence comme suit : «Voici l'événement imprévu qui donna une tournure plus heureuse au destin d'Anton $»^{9}$.

Dans les deux cas, l'événement est inattendu, et il aurait pu ne pas se produire, comme le remarquent Werther dans le roman de Goethe, et le narrateur d'Anton Reiser. La possibilité de ne pas rencontrer Lotte, pour Werther, celle de rester définitivement dans le quatrième état, pour Anton, sont envisagées sérieusement comme l'envers de ce qui arrive effectivement. Cependant, la différence de perspective est sensible d'un roman à l'autre, même si le lecteur de Moritz ne perçoit sans doute pas cette différence, surtout à première lecture. En dépit de sa lourdeur, l'insistance du narrateur sur la futilité et le caractère imprévisible de la circonstance heureuse, sensible par comparaison avec la phrase incidente de Werther, est peu perceptible dans un premier temps. En effet, le lecteur est habitué à ce que de petites causes produisent de grands effets. Il suit volontiers les caprices du hasard, si futiles soient-ils. L'inattendu ne le surprend pas vraiment, et d'une certaine manière, il y est préparé. À une époque où les barrières sociales commencent à tomber, où bien des héros de romans picaresques voient leur fortune se retourner et se trouvent portés par le hasard au sommet de la société, la circonstance fortuite qui va faire accéder Anton aux études n'a rien qui choque la vraisemblance, même si le cas n'est pas entièrement banal. S'il ne se produit rien, Anton risque fort de ne jamais sortir de sa condition d'apprenti, ni de son état. Or, un hasard « providentiel », peut-être le moins probable, compte tenu de la détermination sociale, intervient malgré tout et ménage l'occasion d'un tournant dans la destinée du héros. Il dégage les perspectives d'avenir nécessaires à la poursuite du roman.

Les malheurs du héros semblent prendre fin avec la chance inattendue qui lui est offerte par la rencontre avec le pasteur Marquard. Le lecteur s'attend donc à ce qu'il trouve dans d'autres romans, jusqu'à la limite du

9. Anton Reiser, p. 120 : «Da sich nun für Anton keine solide Aussicht zum Studieren eröffnen wollte, so würde er doch am Ende wahrscheinlich den Entschluß haben fassen müssen, irgendein Handwerk zu lernen, wenn nicht, wider Vermuten, ein sehr geringfügig scheinender Umstand seinem Schicksal in seinem ganzen künftigen Leben eine andere Wendung gegeben hätte "; Anton Reiser, p. 123 : «Der Umstand, wodurch Anton Reisers Schicksal unvermutet eine glücklichere Wendung nahm » (c'est moi qui souligne). Nous citons la traduction de Georges Pauline, Paris, Fayard, 1986 (citée par la suite comme trad.), p. 106, 111. 
vraisemblable qui, nous l'avons vu, n'est pas franchie ici. Il attend le début d'une ascension sociale en rupture avec les vicissitudes qui ont éprouvé l'enfance d'Anton. À cet endroit du récit, le lecteur peut projeter le début du roman de la «carrière " d'Anton qui apparaîtra rétrospectivement comme un parcours programmé, orienté vers un but.

Or, le hasard ne s'insère pas ici dans le cadre de l'enchaînement serré, de la perspective linéaire simple qui est celle du hasard littéraire. Le narrateur épaissit en quelque sorte la situation pour problématiser le hasard. Werther saisit ainsi sans y penser l'occasion de la rencontre avec Lotte, tout aussi naturellement que l'auteur a recours au hasard pour figurer la nécessité littéraire. Il n'en va pas de même dans le roman de Moritz, où le personnage ne peut pas disposer du hasard avec la même facilité.

Tout d'abord, les propos sur la futilité du hasard s'inscrivent directement en contrepoint des plans grandioses et irréalisables échafaudés par Anton. L'accent se trouve donc en partie déplacé vers la vanité des projets du personnage pour s'arracher seul à sa condition d'apprenti. Ses tentatives sont inefficaces et sans prise sur la réalité, alors qu'il suffit au hasard d'un coup de pouce pour retourner la situation. Au cours du roman, c'est ce paradoxe qui ressortira de plus en plus nettement jusqu'à devenir un thème majeur du parcours d'Anton. C'est ainsi que le hasard heureux, qui semble se moquer des efforts démesurés du héros, devient par là même difficile, sinon impossible à lire.

Par ailleurs, ce hasard en définitive heureux ne se présente pas tout d'abord comme tel, mais sous l'aspect d'une malchance. En effet, Anton est surpris par le pasteur à l'instant précis où, engagé dans une bagarre avec des vauriens, il donne de lui une image peu édifiante, susceptible même de ruiner définitivement son crédit auprès du pasteur. C'est pourtant cette rencontre qui attire sur lui l'attention d'un personnage assez puissant pour le sortir de son état. Un tel retournement de situation aurait pu ne pas se produire. Dans d'autres cas semblables, le hasard malheureux ne sera pas transformé en chance : il contribuera à accélérer la chute d'Anton, victime de sa maladresse ou de sa négligence. Or, le lecteur ne le sait pas encore, ce qui ne lui permet pas de relativiser la portée de l'épisode.

Exceptionnellement, Anton parvient à surmonter son sentiment de honte et de mépris de soi pour affronter la situation, non sans avoir traversé une phase de conflit intérieur minutieusement décrite par le narrateur. Le sursaut d'énergie d'Anton est motivé par la force de son désir, qui est d'échapper à sa condition, et la perception claire que le pasteur est l'un des seuls à pouvoir le tirer de son néant ${ }^{10}$. Cet exemple de résolution est suffisamment rare pour être souligné ici.

10. Cf. Anton Reiser, p. 123 : l'expression « mich aus dem Staube hervorzieht » témoigne d'une lucidité peu commune chez Anton, car elle le conduit à décider et à agir. 
Ce n'est donc pas sans difficultés, ni sans accrocs, que le hasard parvient ici à jouer son rôle déterminant. Anton, durant une brève période, est hissé au niveau de ce hasard qu'il a transformé lui-même en chance. Il bénéficie ensuite d'un concours de circonstances qui lève les différents obstacles à son désir d'étudier. Pendant cette brève période, le lecteur est mis en condition d'attendre la suite du « roman » de la bonne fortune du héros qui semble se dessiner. L'intervention du narrateur doué de la connaissance de l'avenir, qui vient relayer un temps la perspective bornée du personnage, entretient ce climat d'expectative. Le narrateur commente : « Sa joie aurait été plus grande encore s'il avait pu savoir que cet incident dû au pur hasard mettrait fin à tous ses soucis et à toutes ses appréhensions et serait le point de départ de son bonheur à venir ${ }^{11}$. Elle conditionne le lecteur à interpréter le hasard comme le fondement (Grundlage) qui semble appeler la construction d'un édifice promis à la stabilité et à la durée, comme le point de départ d'une progression cohérente et linéaire. Si le personnage ne prend pas totalement conscience de sa situation, le narrateur omniscient, lui, marque l'importance de l'événement pour lui et pour le lecteur, ou délègue ce rôle à d'autres personnages qui se font l'écho de ces grandes espérances : «Cette supposition était renforcée par les paroles mystérieuses du desservant de la garnison... "Soyez sur vos gardes et dites-vous bien qu'on vous observe attentivement! De grandes choses se préparent et vous en êtes le centre" " ${ }^{12}$. Le terme de Werk, complétant ici l'image de l'édifice, évoque la dynamique du processus par l'image de la machine mise en cuvre, du hasard opérant dans le sens des intérêts du héros. Lorsque, à la suite de l'intervention du pasteur Marquard, le prince donne un témoignage éclatant de sa faveur à Anton en lui procurant les moyens d'étudier, la Providence est même momentanément convoquée. Dans la vision subjective d'Anton ébloui ${ }^{13}$, elle semble retrouver le rôle d'instance lointaine ou proche qui guide le parcours du héros et le mène à l'accomplissement de son destin. L'atmosphère d'expectative, les promesses d'avenir semblent rompre le cercle vicieux de la détermination psychologique et sociale.

Or, la suite du livre ne répond pas à cette attente, au contraire. Le but à peine entrevu se dérobe, les obstacles s'accumulent sur le chemin d'Anton. Il semble que la circonstance fortuite heureuse, en dépit de ses effets béné-

11. Anton Reiser, p. 124 : « daß dieser ohngefähre Zufall allen seinen ängstlichen Besorgnissen ein Ende machen, und die erste Grundlage seines künftigen Glücks sein würde »; trad., p. 112 (c'est moi qui souligne).

12. Anton Reiser, p. 125 : «Seien Sie ja recht auf Ihrer Hut und denken Sie, daß man genau auf Sie achtgibt. - Es sind große Dinge mit Ihnen im Werke! »; trad., p. 113. Cette prophétie rappelle par son ton mystérieux et son imprécision celles de l'entourage de Heinrich dans la Lebensgeschichte de J. Heinrich Jung-Stilling, mais avec une nuance différente : le ton de menace. Le héros pour lequel la société conçoit des espérances est contraint de ne pas les décevoir, sous peine d'exclusion.

13. Anton Reiser, p. 132 : "Sein Herz ergoß sich wirklich in Dank gegen die Vorsehung. " 
fiques, ne puisse pas jouer le rôle d'un hasard opérant activement. Le mouvement d'ascension à peine ébauché s'interrompt, puis s'inverse, Anton perd peu à peu la position qu'il commençait à conquérir et sa chute, à la fin du livre II, ruine durablement sa première tentative d'intégration dans la société. Les circonstances fortuites de l'existence d'Anton ne ressemblent pas au hasard qui configure fortement le parcours du héros de roman et lui donne un sens. Pour Anton Reiser, et au niveau où il le vit, le hasard ne porte pas, il n'a pas l'épaisseur et la consistance du hasard qui conduit Werther à Lotte et concentre son destin dans cette figure de la nécessité d'une passion fatale. Pour cela, il faudrait que le hasard puisse s'appuyer sur la coopération du héros. Le hasard donne une impulsion, il met en branle, à charge ensuite pour le personnage de l'intégrer en l'interprétant, en se laissant porter par lui. C'est ce que fait Werther en assignant une signification éminente à la rencontre avec Lotte et en orientant sa vie entière autour de sa passion.

Si nous examinons les premières pages du livre II d'Anton Reiser, nous observons que les hasards favorables sont empêchés de produire leurs effets de structuration forte et de configuration nouvelle de l'expérience du héros. Ils sont bien l'occasion d'une «ouverture» (Aussicht) et d'une relance de la dynamique de l'action, mais ils sont presque aussitôt dévoyés par les réactions d'Anton, qui ne sait pas les interpréter correctement ni les utiliser à son profit. Il se révèle incapable, à terme, d'orienter ses efforts avec constance vers le but lointain entrevu. Par ailleurs, ces hasards sont rapidement brouillés par d'autres circonstances qui neutralisent en partie leurs effets positifs. C'est ainsi que le hasard est constamment distrait de son chemin, ses effets positifs amenuisés, son action enrayée par l'activité mentale du héros, affecté par la détermination psychologique originelle et par les effets objectifs de la détermination sociale. Ce sont ces deux points que nous allons examiner.

Différence majeure avec Werther, le narrateur d'Anton Reiser insiste lourdement sur l'impréparation psychologique du personnage, débordé par sa bonne fortune. Pris de stupeur devant un renversement de situation impensable pour lui, l'enfant rejeté, auquel personne jamais n'a accordé la moindre attention ${ }^{14}$, va chercher l'interprétation la plus plausible pour lui, compte tenu de son éducation religieuse, celle de l'élection divine. Nous

14. Le narrateur insiste à dessein sur l'incrédulité d'Anton, que rien n'a préparé à cette soudaine faveur de la fortune, cf. Anton Reiser, p. 131 : «In seinem Leben hatte Anton keine solche Empfindung gehabt, als ihm diese achtungsvolle Begegnung verursachte »; et la répétition littérale des termes comme «Eifersucht der Schulen seinetwegen », "Also Anstalten sollten nur seinetwegen getroffen werden, wegen eines Knaben, den seine eigenen Eltern nicht einmal ihrer Aufimerksamkeit wert gehalten hatten », in Anton Reiser, p. 132, révélatrice de la difficulté, presque acoustique, d'Anton à comprendre que ce discours le concerne personnellement. 
pensons, certes, au Heinrich de Jung-Stilling, en entendant les « prophéties » du desservant de la garnison. Mais le narrateur du roman de Moritz se distancie explicitement de cette lecture providentielle des faits, en insistant sur la composante du délire subjectif. Le hasard ne fournit pas à Anton l'occasion d'agir dans et sur la réalité, il occasionne chez lui un accès de vanité $^{15}$, un échauffement de l'imagination ${ }^{16}$. L'enfant devient le spectateur complaisant de sa nouvelle image de lui-même, tandis que le narrateur s'installe dans le rôle de spectateur critique. D'innombrables exemples montrent dans ces pages denses la réserve du narrateur devant le comportement d'Anton. Le hasard heureux est simple prétexte à des rêveries exaltées qui sont interprétées comme autant de compensations aux souffrances endurées ${ }^{17}$. L'attention du lecteur est volontairement attirée sur la détermination intérieure du héros et sa désadaptation. Au débordement de l'activité imaginatrice correspond la passivité effective.

L'euphorie trompeuse qui dérobe la réalité aux yeux d'Anton ne peut se maintenir lorsqu'un nouveau hasard, cette fois-ci malheureux, vient remettre en cause la supériorité qu'il croyait définitivement acquise. Anton se pensait désormais élu pour occuper la première place, il doit s'effacer à l'arrivée d'un jeune homme de famille aisée. C'est la première allusion au handicap social qui est un des thèmes majeurs du livre II. Cette seconde circonstance fortuite, tout aussi imprévue que la première, détermine chez Anton une nouvelle dérobade et un accès de "la plus noire envie " ${ }^{18}$. Le narrateur présente l'incident comme châtiment mérité de sa « vanité », manière de souligner que le personnage est tout aussi peu préparé que la première fois à ce choc nouveau avec la réalité. On pourrait citer bien des exemples encore de cette épreuve de la déception, dans la période même où le hasard heureux est encore opérant. Le jour de la confirmation, qui représente pour Anton une première apparition publique, et la chance, pense-t-il,

15. Anton Reiser, p. 126 : "sich eine wichtigere Person, als bisher zu glauben », « seine kleine Eitelkeit mehr wie zuviel Nahrung erhielt ".

16. Cf., en part., Anton Reiser, p. 126: «Dies erhitzte seine Einbildungskraft bis auf einen hohen Grad», "alle die schwärmerischen Ideen " (c'est moi qui souligne). Le terme de «schwärmerisch», en relation avec le réveil des impressions religieuses de Braunschweig, est à comprendre dans le cadre du combat de l'Aufklärung contre la «Schwärmerei ». Pour évoquer d'un mot les enjeux, c'est-à-dire la relation au réel, on rappellera ici l'opposition entre la rêverie exaltée (« andächtig schwärmen ») et la bonne action ( "gut handeln ») dans le Nathan der Weise de Lessing, I, 2. Hans-Jürgen Schings analyse la critique éclairée de Moritz (qui culmine dans l'épisode de Lobenstein) dans son ouvrage Melancholie und Aufklärung, Stuttgart, Metzler, 1977. Pour une présentation générale du débat, cf. « Die Aufklärung und die Schwärmer ", dir. Norbert HiNSKE, Aufklärung, Hambourg, Meiner, 1988.

17. Anton Reiser, p. 132 : «das süße Traumbild eines sehnlich gewünschten, aber nie gehofften Glückes, war ehe er es sich versehn, wirklich geworden, und er konnte nun seinen angenehmsten Phantasien nachhängen, ohne zu fürchten, daß er durch Mangel und Armut darin gestört werden würde » (c'est moi qui souligne).

18. Anton Reiser, p. 129 : «Der schwärzeste Neid... », la noirceur introduit le motif de la mélancolie caractéristique d'Anton. 
de jouer les premiers rôles, est assombri par de multiples incidents : 1'occasion perdue de se distinguer, l'absence de l'émotion religieuse conventionnelle, la distance du pasteur Marquard, la froideur de ses hôtes ${ }^{19}$. Alors que la confirmation devait marquer, dans la logique d'un hasard porteur, un premier pas vers l'affirmation de soi et l'intégration sociale — Anton l'aborde avec le sentiment d'un général confiant dans l'issue victorieuse d'une bataille où il se couvrira de gloire - , elle est marquée du signe de la frustration et de l'exclusion.

Les incidents et premiers dysfonctionnements perceptibles dans cette phase encore ascendante viennent pondérer l'appréciation optimiste des effets du hasard. Le début du livre II laissait entrevoir et espérer une mutation décisive dans l'existence du héros. Or, contrairement à ce que le lecteur naïf avait été induit à penser, il s'avère qu'un hasard isolé ne suffit pas à assurer définitivement le bonheur d'Anton. Le personnage éprouve, au quotidien, la nécessité de nouveaux relais pour entretenir et prolonger l'évolution positive qui se dessine. Or, ces relais font justement défaut lorsque le besoin s'en fait sentir. Le pasteur Marquard, pour prendre cet exemple, joue un rôle irremplaçable à un moment crucial. Mais l'assistance qu'il apporte à Anton est insuffisante, parce que ponctuelle. Une fois le " cas Anton » réglé de manière apparemment satisfaisante, il s'éloigne jusqu'à devenir inaccessible, ce qui revient dans les faits à abandonner Anton sans défense dans une société où il n'a pas naturellement sa place. Seule une surveillance attentive et continue serait assez efficace pour entraver le jeu des mécanismes sociaux qui confirment et aggravent le handicap de la naissance dans le quatrième état. Or, de façon générale, l'attention dont bénéficie Anton n'est, de son point de vue, qu'épisodique.

Le hasard initial n'est donc qu'un élément de la résolution du problème posé par l'avenir d'Anton. Dans le contexte du roman psychologique, il ne suffit pas à fonder la nécessité de sa carrière. Il importe peu que se produise un hasard favorable - une chance isolée peut toujours se présenter, même dans une existence aussi défavorisée que celle du héros -, la véritable question est celle de sa portée réelle. Une circonstance heureuse fortuite est inopérante, à terme, lorsqu'elle n'est pas relayée et soutenue par une volonté active (celle d'Anton ou de ses éducateurs), seule susceptible de convertir ce hasard en nécessité littéraire et d'arracher le héros à la contingence de son existence. Faute de quoi, Anton se retrouve à la merci d'autres hasards susceptibles d'infirmer les effets du premier et d'invalider les résultats acquis ${ }^{20}$. Le problème réel se pose donc avec l'inscription dans

19. Anton Reiser, p. 138 sqq.

20. Un exemple intéressant de retournement : pendant la phase d'ascension, les progrès d'Anton s'inscrivent dans la logique d'un programme d'apprentissage qui ne doit rien au hasard (cf. infra, n. 37). Lorsqu'il est abandonné de tous, Anton fournit encore épisodique- 
la durée. C'est ici que le roman est en quelque sorte contraint de refouler la perspective du hasard littéraire, intenable, pour restaurer celle du hasard vécu. Celui-ci ouvre à la figuration romanesque un tout autre champ de réalité : à la cohérence visible d'une carrière linéaire, réglée par une instance narrative ordonnatrice qui maîtrise le hasard et le rend signifiant, se substitue la perspective du flux et du reflux de la vie intérieure, celle de l'entrelacs inextricable des petits faits perçus par la conscience, des hasards qui interviennent brutalement et semblent décourager toute tentative de prendre barre sur la réalité, et par suite, tout effort d'interprétation et de lecture cohérente de ce qui arrive.

Dans le courant du livre II, le revirement est marqué par un incident malheureux qui se transforme en catastrophe irrémédiable, inverse le mouvement d'ascension ébauché et précipite la chute d'Anton. À l'origine, il s'agit d'une simple maladresse : Anton déchire la page de son livre d'examen, ce qui indispose le directeur et paralyse le héros. Peu de temps après, il est surpris à bavarder et accusé publiquement de bassesse morale par le même directeur, qui n'a visiblement pas oublié l'incident. Victime des apparences, incapable de se défendre, Anton se voit ôter définitivement et l'estime du directeur, et la sympathie de ses camarades ${ }^{21}$. Le rapprochement avec l'épisode qui ouvre le livre II fait apparaître que, confronté à deux situations où les apparences sont contre lui, Anton réagit de manière diamétralement opposée. D'un épisode à l'autre, on notera une gradation significative. Au début du livre II, Anton était capable d'un sursaut d'énergie, ici, il est impuissant à retourner la situation. Dans le premier cas, le seul obstacle à vaincre était la détermination psychologique héritée de son enfance malheureuse; dans le second, il s'y ajoute le poids croissant de la détermination sociale dont le personnage fait l'expérience jour après jour. À l'occasion de cet épisode où le hasard intervient une nouvelle fois, le narrateur reconstitue de façon magistrale, pour le lecteur, l'articulation de la détermination intérieure et extérieure qui conjuguent leurs effets pour surdéterminer le hasard ${ }^{22}$. L'incident malheureux produit des effets catas-

ment un travail, mais sans méthode ni continuité. Ses progrès ne sont plus alors que « fortuits », cf. Anton Reiser, p. 211.

21. Anton Reiser, p. 192-194.

22. La figuration de l'enchaînement fatal impose ici de déborder le cadre de la narration strictement chronologique pour rapprocher et comprimer dans le récit les facteurs et circonstances qui ont eu une incidence sur l'issue du conflit, ou qui sont nécessaires à sa pleine intelligibilité pour le lecteur. La relation de l'épisode annoncé est précédé d'une longue introduction où alternent narration et commentaire : l'arrivée d'Anton dans l'établissement et la réaction de curiosité de ses condisciples, qui renvoie Anton à son complexe d'infériorité sociale; la remarque "documentaire " sur la relation entre le classement et la place dans la classe permet de comprendre la relégation d'Anton au dernier rang à la fin de l'épisode. Suit la narration de l'incident proprement dit. Elle est reliée à l'épisode de la page déchirée, quelques pages auparavant, pour expliquer la paralysie d'Anton devant une accusation injuste. La prise en compte des éléments psychologiques se greffe sur la narration des circonstances 
trophiques irréversibles, qui invalident les efforts du héros et font apparaître le hasard du début comme fragile, en définitive. Le héros vit cet épisode comme un châtiment «immérité » (unverdient) et en retire l'impression que la fatalité s'acharne désormais sur lui.

Le premier livre a construit le cadre de la détermination intérieure qui invalide par avance toute tentative de briser l'enfermement défensif dans le monde de l'imagination. Le héros ne dispose pas d'un modèle d'interprétation cohérent ni de possibilités d'ajustement au réel. Le livre II est le récit de la première tentative de socialisation. C'est ici que le hasard intervient dans la destinée d'Anton. On notera que le terme est pratiquement absent du livre I, alors que ses occurrences se multiplient dans le livre II. Or, le hasard, facteur nouveau de l'existence d'Anton, n'est pas traité comme ouverture à l'expérience et à l'interprétation, il ne fonctionne pas comme l'auxiliaire d'un accomplissement positif ou négatif, mais comme révélateur, pour le narrateur et le lecteur, de la détermination conjuguée de l'intérieur et de l'extérieur qui entrave la progression du héros.

On se souvient que Moritz, dans la préface au livre II, souligne l'importance de l'opacité comme expérience première de la réflexion de la conscience. L'individu qui vit dans la confusion de ses perceptions immédiates subit la multiplicité des accidents qui l'affectent de façon contradictoire et, semble-t-il, absurde, sans comprendre la détermination qui les affecte. Le narrateur, qui analyse et explicite la logique de ses comportements et de ses échecs en les ramenant à leurs causes psychologiques et sociales, n'intervient pas cependant pour clarifier et ordonner le cours des événements en nous donnant la raison finale de cet itinéraire tortueux. Il nous présente le héros qui vit au jour le jour la succession des incidents tantôt favorables, tantôt défavorables, le croisement de ce qui sert sa formation, sa «Bildung » - l'attention des professeurs, ses apprentissages et de ce qui invalide ses progrès en freinant son zèle - la charité humiliante, l'absence de foyer. Le héros est élevé, puis précipité dans l'abîme, oscille continuellement entre l'espoir et le désespoir. Le hasard heureux ou malheureux reste pour une large part incompréhensible à Anton, il se dérobe à l'interprétation cohérente. Le personnage ne trouve, en lui-même

objectives, ce qui figure dans la trame même du discours l'interaction des deux séries : par ex., la réaction de curiosité des condisciples d'Anton est présentée objectivement, puis dans la perception subjective - faussée - d'Anton, dont le narrateur décompose la logique d'erreur : l'ignorance du sens exact de «Famulus », qu'il comprend comme l'équivalent d' « esclave », ce qui lui fait sentir sa situation subalterne, complexe d'infériorité renforcé par son apparence physique. La transformation du simple hasard en catastrophe irrémédiable se résume dans la différence d'emploi de l'épithète de «Famulus » à propos d'Anton, au début et à la fin. L'expression "des Rektors Famulus », chuchotée au passage d'Anton, est " objectivement » neutre, elle cerne une réalité objective, situe Anton comme « assistant " du recteur. Après l'incident, Anton est devenu « der Famulus », une épithète que ses condisciples ne chuchotent plus, mais lui jettent à la figure, elle devient injurieuse et dégradante. 
comme dans le monde extérieur, ni repère ni ancrage. Il n'éprouve que son impuissance à maîtriser les hasards, à détourner les coups qui le frappent. $\mathrm{Ce}$ que vit Anton n'est pas récupérable d'une manière ou d'une autre par une instance supérieure ordonnatrice de sa vie, et du roman.

L'image de la fatalité aveugle à la fin du livre II correspond à celle de Providence, au début, comme le pendant négatif de la représentation mentale du personnage. Après l'échec de sa première tentative de socialisation, Anton se retrouve dans un état de délabrement total, réduit à la compagnie d'autres épaves de la société. Il se réfugie dans un jeu infantile : il range des noyaux de cerises et de prunes en ordre de bataille, s'arme d'un marteau et les yeux fermés, il frappe indifféremment les uns et les autres, avant de compter les «morts ${ }^{23}$. En jouant le rôle d'un Dieu méchant acharné à détruire sa création, Anton figure la fatalité aveugle qui le poursuit, c'est la seule possibilité qui lui reste de mettre mentalement son expérience à distance, lors même qu'elle lui reste entièrement opaque ${ }^{24}$.

Le livre II se joue sur l'impossibilité de tenir la perspective du roman qui semblait s'amorcer au début avec l'intervention d'un hasard heureux, l'attente de "grandes choses », le début d'une ascension sociale. Le narrateur qui introduit les circonstances fortuites de nature à changer l'existence du héros en limite dans le même temps la portée, lors même que le héros semble encore engagé dans une évolution positive. Il restitue la densité d'un réel contradictoire en faisant apparaître la difficulté, puis l'impossibilité de situer le héros dans un temps orienté vers une fin, un accomplissement réel, un projet unificateur. Il s'avère qu'aucun hasard n'est assez puissant pour repousser, sinon lever la détermination intérieure et extérieure qui pèse sur le personnage. Celui-ci peut croire un temps, et le lecteur avec lui, que les progrès de l'intégration sociale, le développement intellectuel, en créant les conditions de nouvelles expériences, atténuent les effets néfastes de cette détermination; or, ils ne font que la contourner, sans s'attaquer à ses racines profondes. Le hasard est ici non pas le mode d'apparition de la nécessité littéraire, mais occasion de manifester la puissance des obstacles, le signe de la contingence qui affecte l'existence et l'expérience du héros.

La logique du roman, tel que Blanckenburg en a posé le modèle, se trouve ici subvertie. En effet, selon l'auteur de Versuch über den Roman ${ }^{25}$,

23. Anton Reiser, p. 230.

24. L'horizon de ce problème est la question de la Providence. On se reportera pour cette question au chapitre consacré à l'analyse d'Anton Reiser dans l'ouvrage de Werner Frick, Providenz und Kontingenz. Untersuchungen zur Schicksalssemantik im deutschen und europäischen Roman des 17. und 18. Jahrhunderts, 2 t., Tübingen, Niemeyer, 1989. Opposant le modèle de roman fixé par Blanckenburg et le projet de Moritz, l'auteur prend acte de la totale disparition de l'horizon de la Providence dans Anton Reiser, ce qui représente la négation du modèle religieux qui a dominé l'enfance d'Anton.

25. Fac-similé de l'éd. orig. de 1774, postf. d'Eberhard LÄMMERT, Stuttgart, Metzler, 1965. Pour la comparaison entre le programme tracé dans l'essai de Blanckenburg sur le roman et la 
tout ce qui arrive au héros, les différents facteurs extérieurs tels que l'éducation, la société, la fonction qu'il occupe, doivent le déterminer en dernière instance positivement comme autant de conditions nécessaires à son accomplissement. Le roman produit et conserve l'énergie tout au long du parcours. En ce sens, il n'y a pas de « roman » d'Anton Reiser ${ }^{26}$. L'œuvre ne se donne pas à lire comme la construction d'un parcours exemplaire, orienté vers une fin heureuse représentant le plus haut accomplissement individuel auquel puisse prétendre le héros, c'est-à-dire une perfection humaine relative - un mariage, par exemple. L'histoire intérieure d'Anton Reiser est celle de la déperdition d'énergie, de l'effritement des projets, des fils rompus. On sait que Blanckenburg répugne à envisager que l'auteur du roman - conçu sur le modèle du Dieu leibnizien de la création - puisse faire mourir son personnage. Il n'admet pas, en effet, que le créateur rejette sa créature au néant après l'en avoir tirée. Le suicide n'a de raison que dans la logique d'un caractère que tout pousse à disparaître. Le suicide de Werther serait sans doute acceptable dans cette perspective, comme « acte ». Anton, par contraste, n'a que des velléités suicidaires : il se trouve en équilibre instable au bord de l'eau, se laisse aller, est sauvé par hasard, et au terme de parcours erratiques, se retrouve encore au bord du suicide. Werther vit dans la tension excessive de ses forces ${ }^{27}$, Anton vit dans la déréliction et le sentiment de l'écrasement.

Pour préciser la forme d'une existence sous le signe de la contingence, la structure et l'allure du parcours de l'individu, on se reportera à l'ensemble de fragments réunis sous le titre de Beiträge zur Philosophie des Lebens ${ }^{28}$.

réalisation de Moritz, cf., entre autres, Joseph FÜRNKÄs, Der Ursprung des psychologischen Romans. Karl Philipp Moritz" "Anton Reiser ", Stuttgart, Metzler, 1977.

26. Jean MONDOT insiste également sur ce qu'il appelle la "récusation du romanesque " dans son article "Anton Reiser ou la double subversion », Études germaniques, 50/1, janv.mars 1995 (numéro qui reprend une partie des communications prononcées au colloque de l'université de Paris IV sur "Karl Philipp Moritz et l'autobiographie » en janvier 1995), p. 27-41, loc. cit., p. 38.

27. La «Überspannung» : cf. la lettre $\left(1^{\text {er }}\right.$ livre, lettre du 12 août) dans laquelle Werther relate sa conversation avec Albert sur le suicide. Werther aborde le sujet pour la première fois, et cherche à légitimer le suicide en le présentant non comme un signe de faiblesse ou de lâcheté morale, mais comme une exaltation des forces humaines dans certains états d'exception - l'effort pour se libérer de la tyrannie, pour sauver ses meubles du brasier, pour se venger d'une offense en s'attaquant seul au grand nombre -, donc à des actes glorieux ou incarnant une forme d'héroïsme. Sur la question du suicide, cf. Gerald HARTUNG, «Über den Selbstmord. Eine Grenzbestimmung des anthropologischen Diskurses im 18. Jahrhundert », in Der ganze Mensch : Anthropologie und Literatur im 18. Jahrhundert, DFG-Symposium 1992, éd. Hans Jürgen ScHINGs, Stuttgart/Weimar, Metzler, 1994, p. 33-54.

28. Publiés pour la première fois en 1780, anonymement, sous le titre de Beiträge zur Philosophie des Lebens aus dem Tagebuch eines Freimaurers. Pour l'analyse de ces textes sous l'angle de l'autobiographie comme constitution du sujet, on se reportera à l'article de JeanMarie PAul, «Les autobiographies de Karl Philipp Moritz », dans les actes du colloque sur Karl Philipp Moritz. Anton Reiser. Autobiographie et avènement du sujet, éd. J.-M. PAul, 
On retiendra, entre autres, un passage où Moritz s'élève à des considérations générales sur l'existence :

«Comment cette existence terrestre peut-elle être une fin, puisque tous les hommes, dans l'ensemble, la considèrent non comme un plaisir mais comme un travail. Cette idée de la vie est aussi contenue, me semble-t-il, dans l'expression naturelle que l'on entend si souvent : il faut chercher à faire son chemin dans le monde! On voit la vie comme un voyage pénible, où l'on se fraie laborieusement un chemin entre les ronces qui nous entourent de toutes parts et freinent notre marche. Que de fourrés épais à traverser avant d'arriver à un espace de verdure où l'on puisse se reposer! Et puis voilà que l'on s'enfonce dans un nouveau fourré, jusqu'à ce que l'on finisse par sortir de la forêt et que l'on voie s'ouvrir devant soi la perspective dégagée que l'on avait avant de s'y enfoncer $" 29$.

On opposera la traversée de la forêt de la vie à la marche du pèlerin, progression pénible, mais orientée vers un but, lointain, et cependant toujours présent à l'horizon qu'il illumine de sa transcendance ${ }^{30}$. La traversée de la forêt en est l'exact renversement, l'existence n'y est plus transcendée ou élevée par le but à atteindre. Les réalités vécues, ce sont les obstacles qui freinent la marche et que l'on écarte l'un après l'autre, au prix d'un labeur épuisant. L'individu, totalement absorbé dans son effort, n'a aucune visibilité, son horizon est borné par le prochain fourré à traverser. La vue ne se dégage que lorsque l'on est sorti de la forêt, c'est-à-dire de l'immédiateté de l'existence. La donnée première de la réalité vécue, c'est donc l'immersion dans un fourré qui semble inextricable. Par ailleurs, la remarque désabusée sur laquelle s'achève le parcours jette le doute sur les raisons de l'entreprendre. La traversée est un retour au point de départ, sans que le marcheur retire un bénéfice sensible d'une expérience qui ne fut que pénible.

« Bibliothèque Le Texte et l'Idée », vol. III, Centre de recherches germaniques et scandinaves de l'université de Nancy II, 1994, p. 67-87.

29. K. P. MorITZ, op. cit. supra n. 3, III, «Erfahrung, Sprache, Denken », p. 66 : «Wie kann dies Erdenleben Zweck sein, da es von allen Menschen, im Ganzen genommen, nicht wie ein Vergnügen, sondern wie eine Arbeit betrachtet wird. Diese Vorstellung vom Leben scheint auch in dem so natürlichen Ausdruck zu liegen, den man so oft hört : man muß suchen durch die Welt zu kommen! Man sieht das Leben als eine beschwerliche Reise an, wo man sich mühsam durch die Dornen hindurch drängen $m u \beta$, die von allen Seiten her unsre Schritte aufhalten. Wie oft muß man sich durch dicke Gebüsche durcharbeiten, ehe man einmal wieder auf einen grïnen Platz zum Ausruhen kommen kann! Und denn geht doch immer wieder ein neues Gebuisch an, bis man endlich durch den ganzen Wald hindurch ist, und dann wieder die freie offne Aussicht vor sich hat, die man hatte, ehe man in den Wald hinein kam."

30. On rappellera ici l'ouvrage du puritain John Bunyan, The Pilgrim's Progress, référence littéraire majeure de l'expérience religieuse, d'une influence capitale pour Jung-Stilling, non seulement pour l'autobiographie (cf., entre autres, l'image frontispice du livre Henrich Stillings Jugend, Jünglingsjahre, Wanderschaft und häusliches Leben, éd. Dieter CuNz, Stuttgart, Reclam, p. 305) mais aussi pour le roman allégorique Heimweh. 
Le sens de l'épreuve s'épuise dans l'effort qui mine les forces ${ }^{31}$, sans que l'individu trouve l'occasion de les restaurer, car le temps est uniformément celui du labeur et ne réserve aucune surprise. La vue finale, qui se dégage au moment où les forces sont exténuées, n'est pas le but mérité et attendu, le déploiement d'un panorama sublime qui récompenserait le marcheur, mais l'occasion d'une constatation désolante : ce que l'on voit, c'est ce que l'on a déjà vu; on aurait pu se dispenser de l'épreuve. L'idée même d'une épreuve impliquerait contact, rencontre et transformation productive. Or, celle-ci n'a pas lieu. C'est le non-sens qui menace.

L'expérience du flux et du reflux de la vie mentale, sans prise efficace sur le réel, la perspective métaphysique d'une existence sans finalité propre, vouée à la répétition et à l'insignifiance, forment la donnée première de l'histoire intérieure d'Anton Reiser, comme de celle de Karl Philipp Moritz. Le narrateur du roman nous fait suivre l'histoire d'un personnage qui n'est plus valorisé par un au-delà d'elle-même et se trouve en danger de sombrer dans l'insignifiance de sa singularité isolée. Un épisode de la fin du roman illustrera notre propos, en faisant intervenir encore une fois le hasard. Anton, surpris à cueillir une prune, se fait chasser par le propriétaire auquel il donne son mouchoir en dédommagement. Poursuivant son chemin, il ramasse un couteau qu'il va tenter d'échanger contre son mouchoir. Le propriétaire du jardin, reconnaissant le couteau qu'il avait perdu, se jette au cou d'Anton avec de grandes démonstrations d'amitié et lui dit qu'il doit être un enfant de la Providence :

«En le quittant, Reiser réfléchit à cet étrange incident; il lui parut d'autant plus remarquable que c'était la première fois de sa vie que se produisait en sa faveur un hasard vraiment heureux nécessitant la conjonction de plusieurs circonstances qu'en général on ne trouve pas réunies ${ }^{32}$.

Ces réflexions de Reiser nous livrent le dernier mot de la sagesse du héros concernant le hasard. À ce moment proche de la fin du roman, il ne s'arrête pas à l'interprétation du jardinier convoquant instantanément la Providence qui lui a fait retrouver son couteau. Il ne fait que s'étonner de la conjonction accidentelle de multiples circonstances. De plus, ce hasard se trouve circonscrit dans un mince épisode indifférent à la destinée du héros, il n'a aucun visage signifiant. L'absurdité de ce hasard heureux ressort d'autant plus que, une fois ce moment passé, le sort recommence à s'acharner sur Reiser. Le hasard le favorise stérilement dans les petites choses,

31. «Das Leben ist doch eine Mühe, ein Zwang-", loc. cit. supra n. 29, p. 66.

32. Anton Reiser, p. 472 : « Als Reiser im Weggehen über diesen sonderbaren Zufall nachdachte, fiel er ihm um so mehr auf, weil dies das erstemal in seinem Leben war, daß ihm ein eigentlich glückliches Ereignis begegnete, wobei mehrere Umstände sich vereinigen mußten, die sich sonst selten zu vereinigen pflegen »; trad., p. 391. 
comme par caprice, et lui réserve toutes les infortunes, une fois ce mince crédit épuisé. Le narrateur compare ici le destin de son personnage à celui du vicaire de Wakefield, qui gagne quelques sous aux dés peu de temps avant d'apprendre la banqueroute du marchand à qui il a confié tout son bien $^{33}$. L'image de la chance qui s'épuise comme le crédit renvoie aussi à la situation précaire de Reiser dans la société. Le représentant du «quatrième état » ne peut pas plus compter sur la disponibilité du hasard que sur celle de l'argent, réservés à des personnages plus fortunés. L'existence de Reiser est sous le signe de la contingence radicale, ce qui se traduit dans le registre spatial par la figure de l'errance sans but. L'évolution du héros se décrit comme une série (cf. le terme de «Reihe » employé par Heine) de hasards tantôt heureux, tantôt malheureux, sans que la balance soit égale entre les deux. Le hasard heureux (rencontre, lecture, promotion inespérée, occasion d'attirer l'attention d'un public) peut produire des effets positifs éphémères, il ne cassera pas les déterminations fondamentales contre lesquelles il s'inscrit. Sa base est fragile, sa portée minime. Les hasards malheureux pèsent toujours plus lourd, leur portée est immense parce qu'ils vont dans le sens des déterminations fortes de l'existence du personnage, et agissent comme révélateurs de la fragilité de son assise psychologique et sociale. Ils ont le pouvoir d'anéantir toute amorce de réalisation et de rendre tout effort dérisoire.

À première vue, cette expérience figurée de la contingence ne semble pas donner matière à un roman. Nous avons tenté de montrer comment, dans le livre II, l'auteur inscrit dans la trame du texte tout ensemble l'amorce d'un roman et le refoulement d'une perspective narrative traditionnelle de roman de formation. Nous avons montré également comment l'utilisation et la figuration du hasard interviennent dans ce dispositif : le hasard « littéraire », au sens où il crée la nécessité du destin du personnage en légitimant son existence, ne peut pas s'affirmer, et c'est la perspective du hasard vécu qui s'impose, dans son illisibilité même, jusqu'au modèle global de la fatalité aveugle s'acharnant sur le héros.

Nous mentionnerons au passage, sans développer davantage ces réflexions qui sortent du cadre défini ici, que la solution individuelle à cette déréliction est à chercher dans une dimension de l'existence qui permettrait de se soustraire par instants à la prise de la fatalité ou de la totale détermination en conférant une consistance à la vie intérieure de l'individu. Moritz l'envisage dans ses Beiträge : nous retiendrons une phrase particulièrement significative à cet égard. Moritz déclare au terme d'une journée : « Je suis

33. Anton Reiser, p. 472 : «Es war, wie bei dem Landprediger von Wakefield, der einen ganz ungewöhnlichen glücklichen Wurf mit den Würfeln tat, indem er mit seinem Freund um wenige Pfennige spielte, kurz vorher, ehe er die Nachricht von dem Bankrott des Kaufmannes erhielt, durch welche er sein ganzes Vermögen verlor." 
content de cette journée, car je l'ai employée et j'en ai joui ${ }^{34}$. Les deux dimensions de « l'emploi » et du « plaisir » sont essentielles et complémentaires, elles instaurent un temps différent de celui de la frustration et de l'illisibilité, par l'adéquation réalisée entre l'intérieur et l'extérieur. On retrouve dans le roman, par instants fugitifs de plénitude, cette qualité d'expérience, qui laisse la trace du souvenir actuel, présent dans la conscience d'aujourd'hui, comme repère ponctuel, maillon de la chaîne des souvenirs ${ }^{35}$. L'expérience de la lecture ${ }^{36}$, la lecture de Shakespeare, transformée en véritable Erlebnis par le partage avec l'ami ${ }^{37}$, représentent de semblables moments. La stimulation des facultés de l'individu orientées vers la constitution d'un projet permet aussi d'échapper à l'enfermement et à la vision de l'anéantissement, en suggérant une prise possible sur le réel, et en dégageant des «perspectives ${ }^{38}$.

C'est paradoxalement en revendiquant l'extrême particularité d'une existence qui n'est sauvée par aucune instance transcendante que Moritz peut

34. Cf. op. cit. supra n. 29 , p. 63 : " Ich bin zufrieden mit diesem Tage, weil ich ihn angewandt und genossen habe $»$ (c'est moi qui souligne). Le terme de angewandt (employé) ne se confond pas avec la notion d'utilité et de profit (Nutzen), chère à une certaine pensée de l'Aufklärung, dont Moritz critique l'utilitarisme. Moritz, à la suite de Rousseau, défend la finalité propre de l'individu « en soi $\%$, irréductible à la seule fonction de rouage dans la société.

35. Anton Reiser, p. 269 : «Er fühlte, daß sich das Dasein nur an der Kette dieser ununter. brochenen Erinnerungen festhielt. 》

36. Anton Reiser, p. 15. Le passage au présent est suffisamment rare dans le roman pour être signalé : «Mit innigem Vergnügen erinnert er sich noch jetzt an die lebhafte Freude, die er damals genoß $\gg$ (c'est moi qui souligne).

37. Cf. Anton Reiser, p. 267,269 , où l'on remarque encore le passage exceptionnel au présent : "Diese Shakespearenächte gehören zu den angenehmsten Erinnerungen in Reisers Leben."

38. Dans les Beiträge, ce sont le travail et l'effort qui rendent la vie supportable (« Mühe und Arbeit allein macht uns das Leben - erträglich », op. cit. supra n. 29, p. 67). L'imagination reste un leurre, car elle ne permet pas d'échapper aux coups du hasard; au contraire, elle fragilise l'individu en effaçant les exigences de la relation à la réalité. Un rôle éminent revient à toute forme d'apprentissage, de travail intellectuel, philosophique ou poétique, comme exercice de la «Denkkraft", de la faculté de penser autonome et créatrice. On citera ici le passage remarquable des débuts de l'apprentissage philosophique, au début du livre III, p. 252 sqq. Dans le temps où Anton étudie le système philosophique leibnizo-wolffien, les images et le lexique évoquent les idées de progression, de construction, l'activité mentale échappe au hasard, et arrache l'existence d'Anton au hasard. Le héros, livré à lui-même, révèle des capacités et un acharnement étonnants et parvient à une compréhension du système nettement supérieure au niveau scolaire. L'exercice de la pensée peut réaliser la mise à distance de l'expérience immédiate, mais ses limites sont aussi celles de la spéculation abstraite. L'individu, élevé par la réflexion philosophique, se retrouve à terme suspendu au-dessus de l'abîme, confronté à la fatalité aveugle, le «blindes Ohngefähr» qui terrifiait Jung-Stilling dans sa réflexion sur le fondement de sa Lebensgeschichte. Anton cherche à se poser métaphysiquement comme sujet, or dans un système essentiellement logique, comme l'est la philosophie leibnizo-wolffienne, le sujet est une unité vide, il est volatile. L'idée de Dieu lui permet de se penser, d'approcher l'idée d'une permanence abstraite, mais le défaut d'une Providence personnelle souligne la contingence vertigineuse de l'existence individuelle, cf. Anton Reiser, p. 269. 
franchir les obstacles qui le séparent du « roman » dans sa dimension signifiante. Selon nous, c'est là l'enjeu de tout le livre II, qui figure l'horizon du non-sens et le maintient comme tel. À l'appui de notre thèse, nous nous reporterons à la préface du livre III. Si nous suivons l'auteur, nous devons considérer que le «roman » commence véritablement avec les pérégrinations d'Anton Reiser. Fait remarquable, c'est à ce moment que l'auteur, pour la première fois, nomme son personnage. Il ne l'a pas fait dans les deux premières préfaces, et c'est une singularité, déconcertante au premier abord, dont il nous faut tenter de rendre compte. Jusqu'à présent, Anton Reiser existe bien, mais plutôt, semble-t-il, comme sujet d'expérimentation psychologique, individu particulier destiné à être observé dans l'ensemble des déterminations qui le constituent en identité négative : échec de la constitution d'un moi assuré, échec de l'intégration dans la société. À la fin du livre II, la perspective de l'histoire intérieure, (auto-)biographique, est suffisamment affirmée contre la tentation de faire un « roman » superficiellement intéressant par la multiplicité des événements et des personnages ${ }^{39}$. Les petits faits, les hasards vécus tissent la trame fondamentale d'une histoire dont le sens est à chercher dans l'irréductible singularité et la confusion d'une expérience individuelle. C'est alors la perception progressive d'une consistance intérieure qui ménage l'accès à l'interprétation, et donc à la possible généralisation d'une expérience humaine. La visée psychologique et pédagogique déjà présente s'affirme, en même temps que Moritz donne enfin à son personnage la consistance d'un personnage de « roman " doué d'une existence propre, destiné à parcourir un trajet, celui de l'errance et de l'aveuglement. C'est à partir du hasard vécu, dans sa confusion même, que le narrateur, et derrière lui l'auteur, formulent la loi d'une expérience. L'existence particulière d'Anton, dans le sentiment de sa contingence, est reliée à la possibilité d'une signification, en l'absence d'un horizon providentiel. À partir du livre III commence donc l'élaboration du " roman » proprement dit, c'est-à-dire la figuration d'un parcours donné en exemple, et dont la formule générale se retrouve, résumée en quelques phrases énergiques, dans la préface au livre IV : constat de la double détermination qui marque définitivement le personnage, et constat d'échec de la fausse vocation poétique et théâtrale. Mais par le biais de la perspective du psychologue, qui élabore ses modèles d'interprétation et ses normes

39. On connâ̂t le propos de Moritz, présentant son Magazin für Erfahrungsseelenkunde en spécifiant qu'il ne s'agissait « ni de roman, ni de théâtre, ni de verbiage moral » ( Kein Roman, kein Theater, und kein moralisches Geschwätz»). Le roman ne parviendrait, selon K. P. MoRITZ, op. cit. supra n. 3, III, p. 92, dans ses Aussichten zu einer Experimentalseelenlehre, qu'à appréhender la superficie d'une histoire dont il faut explorer la profondeur. Moritz emploie à ce propos l'image du mécanisme d'une horloge, dont le romancier ne verrait que les aiguilles, alors que le spécialiste de l'introspection viserait à voir l'intérieur, les rouages derrière le cadran. 
morales à partir des individus, Anton Reiser se retrouve réintégré dans l'humanité, lui, l'individu des comportements apparemment anormaux et asociaux. En formulant les lois de l'expérience d'Anton, le narrateur se trouve d'ailleurs souvent amené à construire des hypothèses sur le modèle : « Si ses parents, ses éducateurs, son entourage... lui avaient accordé plus d'amour, d'attention et de soins..., l'évolution d'Anton n'aurait pas été telle. " Cette tournure fréquente nous semble particulièrement intéressante comme symétrique inverse d'une autre tournure, tout aussi fréquente, qui correspond à la perspective du hasard vécu, «Es fügte sich... ». L'événement qui arrive dans la vie d'Anton est un accident, fruit d'une conjoncture opaque, mais l'analyse de ces accidents, reliés à d'autres analogues, permet d'élaborer un modèle causal, « cette cause devait produire cet effet », et de construire une hypothèse alternative. "Si l'on avait pu supprimer cette cause, ces effets ne se seraient pas produits. » Dans un monde d'où la Providence est absente, le hasard vécu est une catégorie essentielle de l'existence individuelle, le psychologue ici est la seule instance qui puisse reprendre ce qui est vécu comme hasard par l'individu et créer les conditions de la figuration d'une identité par la connaissance de ses déterminations.

On voit dès lors que la remise en cause du roman traditionnel amène l'auteur à dégager l'espace d'une autre expérience, l'expérience psychologique du flux et du reflux de la vie intérieure. Celle-ci non seulement fournit un nouveau matériau au romancier, mais aussi induit l'élaboration d'une nouvelle forme de figuration de cette expérience, centrée sur l'appréhension de la consistance de la vie intérieure qui, à défaut de trouver des héritiers directs en son temps, éveille des échos profonds dans le nôtre ${ }^{40}$. Si Anton Reiser ne figure pas un «destin d'époque » au sens de celui de Werther, le héros tragique auquel s'identifie une génération entière, il est bien l'emblème d'une autre modernité.

Anne LaGNY (juin 1996).

40. Nous n'en voulons pour preuve que la relecture de Moritz par Arno Schmid, « Die Schreckensmänner/Karl Philipp Moritz zum 200. Geburtstag ", in Dya na Sore. Gespräche in einer Bibliothek, repr. der von Arno Schmidt autorisierten Erstausgabe, Karlsruhe, Stahlberg Verlag, 1958, Francfort-sur-le-Main, Fischer, 1989, p. 356-390. 